\title{
Geómetras en el paraíso: El levantamiento topográfico del Real Sitio de Riofrío (1868-69) ${ }^{1}$
}

\author{
José Luis URTEAGA GONZÁLEZ \\ Universidad de Barcelona \\ urteaga@ub.edu \\ Concepción CAMARERO BULLÓN \\ Universidad Autónoma de Madrid \\ concepción.camarero@uam.es
}

Recibido: 17 de febrero de 2014

Enviado a evaluar: 3 de mayo de 2014

Aceptado: de mayo de 2014

\section{RESUMEN}

En agosto de 1868 un equipo de geómetras de la Junta General de Estadística, dirigido por José Giralt Torner, inició los trabajos de campo necesarios para proceder a una representación cartográfica fiable del Real Sitio de Riofrío (Segovia). El levantamiento formaba parte de un proyecto más ambicioso, que tenía por objeto el inventario y deslinde del patrimonio territorial de la Corona. Los planos formados por los geómetras de la Junta de Estadística, que se conservan inéditos en el archivo del Instituto Geográfico Nacional, constituyen un conjunto documental de notable valor geohistório, sin posible parangón entre las fuentes iconográficas del siglo XIX. Este artículo, que se apoya esencialmente en fuentes primarias, da cuenta de la cartografía conservada, describe la técnica de levantamiento, e identifica a los protagonistas de tales trabajos.

Palabras clave: Real Sitio de Riofrío, cartografía, Junta General de Estadística, José Giralt Torner.

${ }^{1}$ Este trabajo se ha realizado en el marco de los proyectos de investigación CSO2011-29027C02-01 y CSO2011-29027-C02-02, financiados por la Dirección General de Investigación del Ministerio de Ciencia e Innovación. Los autores queremos expresar nuestra gratitud a Andrés Arístegui, Francisco Dávila, Esteban Escolano, Carmina Rimón y Enrique Rojo, del Instituto Geográfico Nacional (IGN), por la ayuda prestada en la localización de los fondos cartográficos que constituyen la base de este trabajo. 


\title{
Land surveyors in paradise: the topographical survey of the Royal Site of Riofrío (Spain), 1868-1869
}

\begin{abstract}
In August 1868 a team of surveyors from the General Statistics Board of Spain ("Junta General de Estadística"), led by José Giralt Torner, began field work needed to produce a reliable mapping of the Royal Site of Riofrío (Segovia, Spain). The survey was part of a more ambitious project, which aimed the inventory and demarcation of the Royal Heritage. Maps formed by the surveyors of the General Statistics Board, which are preserved in the archive of the National Geographic Institute, are an irreplaceable historical source, without possible comparison with the iconographic sources of the nineteenth century. This paper, which is based basically on primary sources, report on the preserved cartography, describes the survey system, and identify the protagonists of these works.
\end{abstract}

Key words: Royal Site of Riofrío, cartography, General Statistics Board of Spain, José Giralt Torner.

\author{
Géomètres au paradis: la levé topographique \\ du Palais Royal de Riofrío (Espagne), 1868-1869
}

\section{RÉSUMÉ}

En Août 1868, une équipe de cartographes du Conseil général de la statistique de l'Espagne ("Junta General de Estadística"), dirigé par José Giralt Torner, a commencé à travailler sur le terrain pour effectuer une cartographie fiable du Palais Royal de Riofrío (Real Sitio de Riofrío, Segovia). Le travail faisait partie d'un projet plus ambitieux, qui vise à l'inventaire et à la démarcation des terres appartenant à la Couronne. Les cartes formées par les experts du Conseil général de la statistique, qui sont conservés dans les archives de l'Institut Géographique National, sont un ensemble de valeur documentaire remarquable, sans comparaison possible avec les sources iconographiques du XIXe siècle. Cet article, qui se fonde essentiellement sur des sources primaires, décrit la cartographie conservée et identifie les protagonistes de ces œuvres.

Mots clés: Palais Royal de Riofrío, cartographie, Junta General de Estadística, José Giralt Torner.

\section{INTRODUCCIÓN}

El 25 de julio de 1868 José Almirante Torroella, jefe de la sección de trabajos catastrales de la Junta General de Estadística, decidió acometer el levantamiento topográfico de las posesiones pertenecientes al patrimonio de la Corona en el Real Sitio de San Ildefonso (Segovia), entre las cuales figuraba el Real bosque de Riofrío. Con ello se venía a dar cumplimiento a la Ley de deslinde del Patrimonio de la Corona, aprobada en mayo de 1865 , que ordenaba inventariar y cartografiar la totalidad de las fincas rústicas, palacios y jardines pertenecientes a los Reales Sitios.

No era la primera vez que los cartógrafos visitaban el bosque de Riofrío, pero si la primera ocasión en la que se iba a efectuar un levantamiento sistemático a gran escala, con garantías de precisión tanto de la planimetría como de la altimetría. Pese a su reducida extensión, de poco más de 600 ha, este levantamiento tiene un especial interés por al menos tres motivos. En primer lugar, los planos documentan con gran 
detalle el estado de Riofrío en una coyuntura histórica particularmente interesante: la de la Revolución democrática de 1868. En segundo lugar, la documentación que se conserva del levantamiento, que es de una extraordinaria riqueza, permite analizar las rutinas del trabajo de los geómetras, tanto en el campo como en gabinete, e identificar el instrumental utilizado; en definitiva, Riofrío constituye un ejemplo paradigmático de la práctica topográfico-catastral de la Junta de Estadística a mediados del ochocientos. Por último, pese al esfuerzo erudito realizado en los últimos años, que ha esclarecido numerosos aspectos de la génesis y evolución del Real Sitio (Hernández Cordero, 1995, 1997, 2008, 2009 y 2010; Lavalle Cobo, 1994 y 2002; Sancho, 1996; Sancho y Aparicio, 2012), la planimetría a la que aludimos permanece desconocida para los estudiosos.

Este trabajo se divide en cuatro partes. La primera ofrece una breve descripción geohistórica del bosque de Riofrío, y de su estado a mediados del siglo XIX; en la segunda se identifica a los protagonistas del levantamiento, brindando una breve nota de su trayectoria profesional; en la tercera se presentan los trabajos campo acometidos en el Real Sitio durante el verano y el otoño de 1868; por último, en la cuarta se exponen las operaciones de gabinete realizadas con posterioridad en los talleres de la Junta General de Estadística radicados en Madrid, que se prolongaron hasta el mes de febrero de 1869. Cierran el trabajo unas conclusiones y la relación de la bibliografía consultada.

\section{LA FORMACIÓN DEL REAL SITIO DE RIOFRÍO}

El bosque de Riofrío fue una de las últimas posesiones en incorporarse al Real Patrimonio de la Corona, y la última en ser cartografiada por la Junta General de Estadística $^{2}$. El término, situado cinco $\mathrm{km}$ al sur de Segovia, y trece al oeste de San Ildefonso, pertenecía a Joseph Orovio Recalde y Bravo de Mendoza, hijo del Marqués de Paredes y miembro de una influyente familia segoviana. En 1724 el rey Felipe V (1683-1746) decidió tomarlo en arrendamiento, por la cantidad de 7.000 reales anuales, para destinarlo a coto de caza. De relieve ondulado y suave, la dehesa de Riofrío era ciertamente un lugar ideal para la caza mayor. Estaba poblada de encinas y enebros, y en sus abundantes pastos pronto iba a crecer una nutrida población de gamos, venados y jabalíes, protegida por los guardas de la casa real (Breñosa y Castellarnau, 2884). Un verdadero paraíso cinegético.

${ }^{2}$ Sobre el proceso de levantamiento de los Sitios Reales acometido por la Junta General de Estadística, puede verse Urteaga y Camarero, 2014a. Acerca de la historia de la citada institución puede consultarse Muro, Nadal y Urteaga, 1996. 
A la muerte de Felipe V, en 1746, su viuda la italiana Isabel de Farnesio (16921766) tomó la decisión de construir un palacio para su exclusiva residencia y la de su hijo menor, el infante cardenal don Luis $(1727-1785)^{3}$. De ahí nació el proyecto de transformar el coto de caza en un Real Sitio, cuyo diseño general fue encomendado al arquitecto italiano Vigilio Rabaglio. Tras lograr una autorización del rey Fernando VI (1713-1759), preceptiva por tratarse de un mayorazgo, Isabel de Farnesio compró al Marqués de Paredes la dehesa en 1751, por la cantidad de 783.331 reales de vellón. Paralelamente, el rey entregó a la viuda de Felipe V la jurisdicción civil y criminal sobre todo el término. Con objeto de aumentar la extensión del bosque fueron adquiridas al año siguiente dos fincas vecinas, pertenecientes al Concejo de las Navas y al cura de la localidad, que sumaban unas 36 hectáreas de extensión (Hernando Cordero, 2008). En una de ellas, que tenía un altonazo con buena perspectiva, iba a asentarse la residencia palatina (figura 1).

Figura 1. La dehesa y el palacio de Riofrío, enclavado en uno de los puntos más elevados del término.

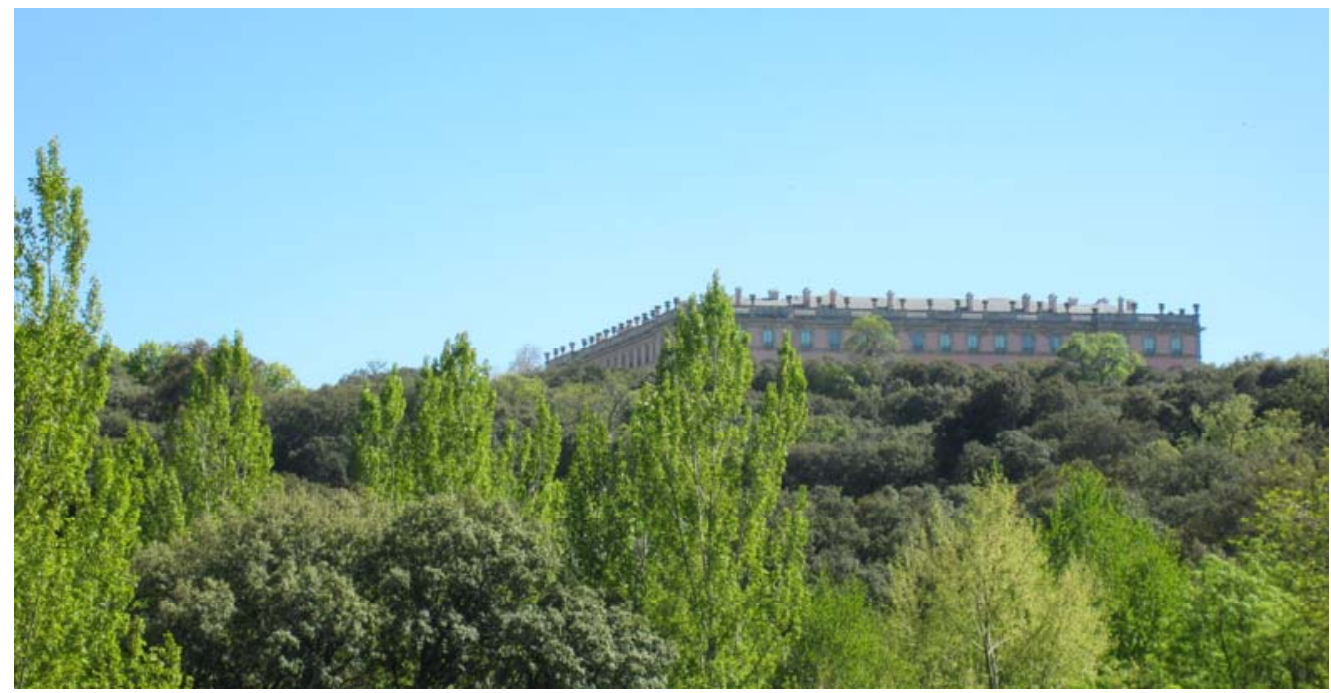

Fuente: Fotografía de los autores, mayo de 2013.

El conjunto del Real Sitio fue cercado mediante una tapia perimetral de mampostería durante el reinado de Carlos IV (1748-1819). Aprovechando la construcción de la cerca, que se levantó entre 1792 y 1795, se produjo una pequeña ampliación territorial de la posesión, mediante compra o permuta de varias fincas

${ }^{3}$ Sobre las motivaciones de Isabel de Farnesio para construir un nuevo complejo palatino en Riofrío debe consultarse Hernando Cordero, 2008, 53 y ss. 
vecinas, que tenían la finalidad esencial de regularizar los límites del bosque. Simultáneamente, se efectuaron algunas mejoras consistentes en la construcción de varios puentes sobre el río Frío, que atraviesa la dehesa de noroeste a sureste, y la colocación de puertas cerradas con verjas de hierro en los accesos a la propiedad.

A mediados del siglo XIX, durante el reinado de Isabel II (1830-1904), el rey consorte Francisco de Asís María Fernando de Borbón (1822-1902) decidió amueblar y habilitar algunas estancias del palacio de Riofrío, para emplearlas como lugar de descanso en las jornadas de cacería. Fuese debido al interés personal de Francisco de Asís por Riofrío, o a algún otro motivo que desconocemos, en 1862 la reina decidió una nueva ampliación del Real Sitio mediante compra de dos fincas colindantes, denominadas el Barrial o la Umbría, y el Chaparral o la Solana. Ambas propiedades sumaban 75 fanegas de extensión, y costaron 55.600 reales $^{4}$.

Con esta adquisición el Real Sitio alcanzó una superficie total de 634 hectáreas, las mismas que tiene en la actualidad ya que sus límites no han sufrido variación desde entonces. La ampliación de 1862 requirió el derribo de más de un km de tapial, y la construcción de una nueva cerca en el límite meridional del bosque, labor que estaba ya concluida en 1868, cuando llegaron al lugar los geómetras de la Junta de Estadística. Según el testimonio de éstos, por entonces residían en el Real Sitio 50 personas, la mayor parte empleados del Patrimonio Real. La caza era abundante, calculándose la presencia de 3.000 cabezas entre venados, gamos y jabalíes. Además de la caza, la única actividad económica era el carboneo, aprovechándose para ello las cortas del encinar ${ }^{5}$. Pocos años más tarde, en 1884, los ingenieros de montes Rafael Breñosa y Joaquín María Castellarnau, que estaban encargados de la gestión del patrimonio forestal del Real Sitio de San Ildefonso, hicieron una estimación cuantitativa del estado del bosque de Riofrío. Según sus cálculos, la especie dominante era la encina, que ocupaba un $80 \%$ de la superficie de la dehesa. El espacio restante estaba poblado por enebros (un 7\% de la superficie), por fresnos y álamos (un 3\%), o despoblado (Breñosa y Castellarnau, 1884). El Real Sitio de Riofrío no se vio afectado por el proceso desamortizador llevado a término durante el Sexenio democrático, y conservó su carácter de coto de caza hasta el siglo XX. En la actualidad es un espacio protegido gestionado por el Patrimonio Nacional. Las encinas siguen dominando su hermoso paisaje (figura 2).

La cartografía de Riofrío anterior al levantamiento de la Junta de Estadística es relativamente pobre. Según Juan Francisco Hernando Cordero, el primer plano detallado del término fue levantado por Vigilio Rabaglio en $1751^{6}$, justo cuando se formalizó la compra de la propiedad por parte de Isabel de Farnesio (Hernando

\footnotetext{
${ }^{4}$ Los detalles de la compra en Hernando Cordero, 2008, 101.

${ }^{5}$ Memoria descriptiva del Sitio de Riofrío. José Giralt Torner, Madrid, 4 de febrero de 1869. Ms. IGN, Archivo Topográfico. Riofrío, Caja 1.

${ }^{6}$ Plano de la Dehesa y Término de Riofrío. Virgilio Rabaglio, 1751. Academia de Bellas Artes de San Fernando, colección Rabaglio, RBG/P 29.
} 
Cordero, 2008). En 1792, al tiempo que se procedía a la construcción de la cerca ordenada por Carlos IV, un artista anónimo ejecutó un nuevo levantamiento cartográfico cuyo original se conserva en el archivo del Palacio Real de Madrid ${ }^{7}$. Según la información que hemos podido reunir no hubo nuevos trabajos topográficos en la primera mitad del siglo XIX. En cualquier caso, los geómetras de la Junta de Estadística ignoraron los ensayos precedentes, y acometieron el levantamiento por sus propios medios. Es hora de presentarles.

Figura 2. Al igual que en el siglo XIX, el bosque de Riofrío sigue siendo hoy, en esencia, una dehesa poblada de encinas.

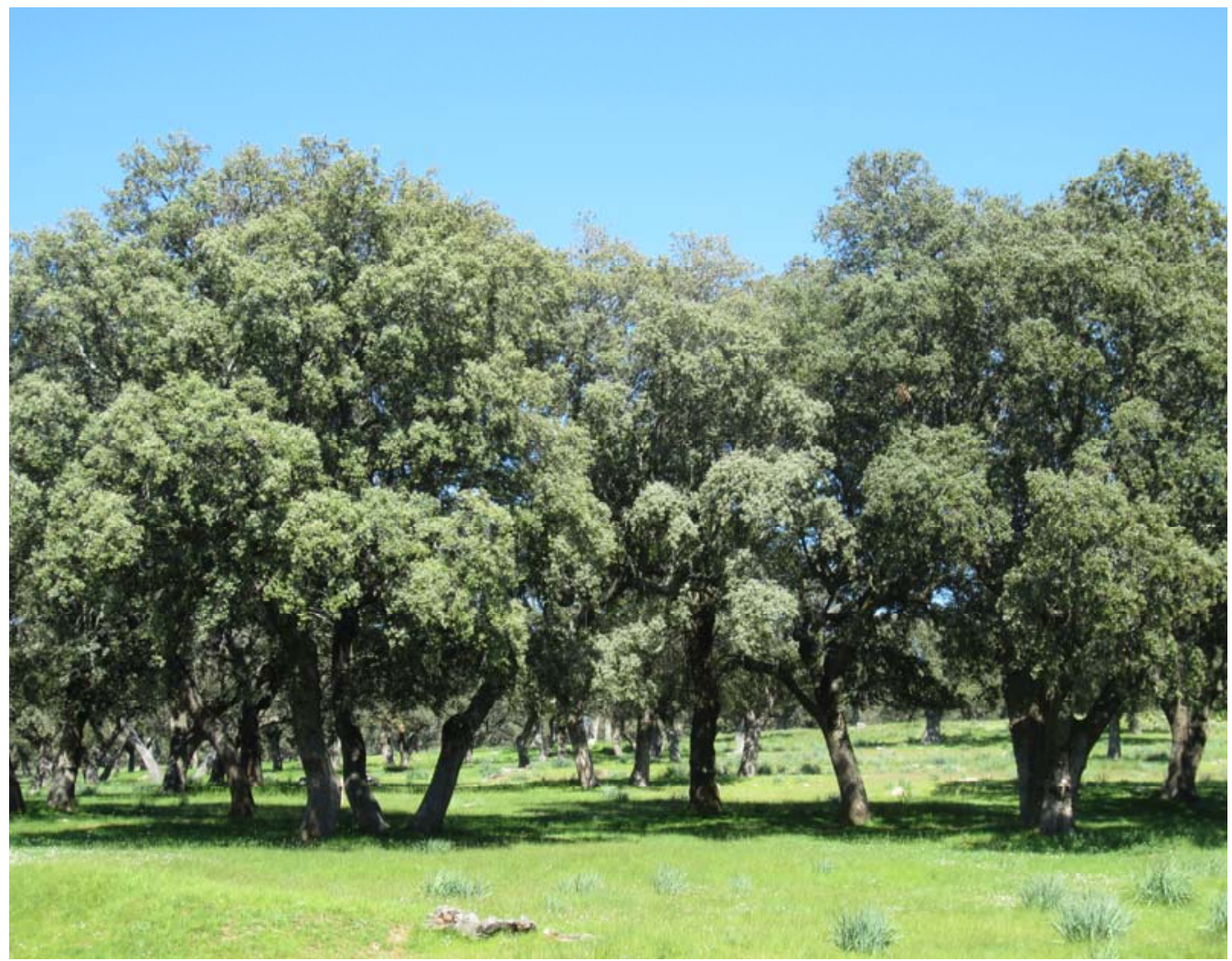

Fuente: Fotografía de los autores, mayo de 2013.

${ }^{7}$ Plano general del Real Sitio y Monte de Riofrío. Nota: En 11 de marzo de 1792 se principió el cercado del Monte reynando D. Carlos IV. Sin autor. Sin fecha. Escala de 5.000 pies castellanos. Aguada en color sobre cartulina. Mal estado de conservación. Una hoja de 81 x 55 cm. Archivo del Palacio Real, Sig. 1.671. 


\section{LOS PROTAGONISTAS DEL LEVANTAMIENTO}

Con la división municipal ochocentista, el Real Sitio de Riofrío había quedado como un anejo del ayuntamiento de San Ildefonso. En consecuencia, su levantamiento topográfico corrió a cargo de la misma brigada enviada a La Granja (Urteaga y Camarero, 2014b). El 28 de agosto de 1868 Ventura Pizcueta Chirivella, jefe de la brigada, nombró una sección integrada por José Giralt Torner, Felipe Casares Moraleda y Domingo Ramos Rodríguez, para que procediera a efectuar los trabajos de campo. La dirección de los trabajos se le confió a José Giralt ${ }^{8}$, el más joven de los tres.

José Giralt Torner había nacido en Sarrià (Barcelona) el 9 de junio de $1842^{9}$. Era hijo de José Giralt, maestro de obras, y de María Torner, ambos barceloneses. Ingresó en la Escuela de Topografía Catastral en octubre de 1864, y siguió un programa de estudios de tres años de duración, durante la etapa más estable y fructífera de este centro. En esos tres cursos siguió un plan de trabajo que buscaba un equilibrio entre la enseñanza práctica y un conocimiento aceptable de los fundamentos teóricos de la cartografía, y que incluía asignaturas específicas de trigonometría rectilínea y esférica, dibujo lineal y del paisaje, topografía, geología y geodesia. Entre sus profesores cabe citar a los ingenieros militares Juan Ruiz Moreno y Eduardo Álvarez García, al dibujante José Pilar Morales, y al geólogo Juan Vilanova Piera (Urteaga, 2011). Terminó sus estudios en diciembre de 1867, obteniendo el número nueve de su promoción. Uno de sus primeros trabajos como geómetra fue precisamente la dirección del levantamiento del Real Sitio de Riofrío.

Sus ayudantes le superaban en experiencia, aunque carecían de su preparación técnica. Domingo Ramos Rodríguez, el más veterano, había nacido en Colomera (Granada) el 4 de agosto de $1829^{10}$. Era hijo de Patricio Ramos y María Antonia Rodríguez, naturales ambos de aquella población andaluza. En agosto de 1861 fue nombrado "portamira aventajado" de la Junta General de Estadística, con el haber de quince reales diarios. Entre 1861 y 1866 realizó trabajos de parcelación en los términos municipales de Fuencarral, Alcobendas y San Sebastián de los Reyes correspondientes a la provincia de Madrid. En 1866, siendo ya parcelador, su carrera profesional estuvo a punto de torcerse: fue expedientado y suspendido de empleo y sueldo por haber vendido planos de la Junta de Estadística a distintos propietarios, algo que estaba estrictamente prohibido. El 10 de julio de 1866 se le dio de baja en la Junta. Debió ser un trago bien amargo. Domingo Ramos estaba casado y tenía tres hijos. Por fortuna para todos ellos, tras presentar un recurso, fue repuesto en el

${ }^{8}$ Memoria descriptiva del Sitio de Riofrío. José Giralt Torner, Madrid, 4 de febrero de 1869. Ms. IGN, Archivo Topográfico. Riofrío, Caja 1.

${ }^{9}$ Archivo histórico y administrativo del IGN. Expediente personal de José Giralt Torner.

${ }^{10}$ Archivo histórico y administrativo del IGN. Expediente personal de Domingo Ramos Rodríguez. 
empleo de parcelador el 12 de enero de 1867. En noviembre de ese mismo año fue destinado al levantamiento del plano urbano de Granada (Camarero et al., 2012), donde permaneció varios meses llegando a firmar una treintena de planos. El 22 de julio de 1868 recibió el nombramiento de ayudante geómetra, con un sueldo anual de 500 escudos, y casi de inmediato fue destinado a Segovia formando parte de la sección encargada de levantamiento del Real Sitio de Riofrío.

Felipe Casares Morales, el segundo ayudante, nació en 1840, y era natural de Nambroca (Toledo) ${ }^{11}$. Había ingresado como peón temporero en la Junta General de Estadística y, al igual que Domingo Ramos, el 14 de septiembre de 1861 fue nombrado portamira aventajado por el Vicepresidente de la Junta. Los portamiras podían seguir estudios en la Escuela de Topografía Catastral, aunque limitándose a un solo curso. Si finalizaban con provecho ese año de estudios, que incluía enseñanzas de dibujo lineal, geología, geografía y dibujo topográfico, recibían el nombramiento de parceladores. Ese fue el camino elegido por Felipe Casares para avanzar en su carrera. El 22 de julio de 1868 fue nombrado ayudante geómetra, y casi de inmediato partió para tierras segovianas.

Giralt y sus colaboradores se desplazaron a Riofrío el 24 de agosto de 1868, iniciando de inmediato los trabajos de campo con el auxilio de varios guardias y peones del Real Sitio, que eran retribuidos por el Patrimonio Real. Los trabajos de campo, que se describen a continuación, se prolongaron durante mes y medio ${ }^{12}$.

\section{TRABAJOS DE CAMPO}

Un aspecto relevante del levantamiento efectuado en Riofrío es que fue realizado siguiendo los requisitos y formalidades previstas en el Reglamento general de operaciones topográfico-catastrales aprobado por la Junta de Estadística en 1865. De acuerdo con lo estipulado en este reglamento, los trabajos debían dividirse en cinco etapas: 1) reconocimiento del perímetro del término y señalamiento de límites de las propiedades, 2) operaciones trigonométricas, 3) levantamiento de detalle, 4) trabajos de gabinete, y 5) formación de listas de propietarios y cédulas catastrales. En el caso del Real Sitio de Riofrío la última de las operaciones era superflua, ya que había un único propietario; la primera era a todas luces sencilla, ya que la propiedad estaba cercada con un muro perimetral.

Giralt se hizo cargo personalmente de la triangulación topográfica del término y de su poligonación, empleando como instrumento un teodolito de Brunner. La operación se realizó siguiendo el perímetro de la cerca, midiendo los ejes dos veces y refiriéndolos al muro perimetral. Algunos puntos de esta poligonación se consideraron vértices de una pequeña triangulación, que quedó enlazada con la de

${ }^{11}$ Archivo histórico y administrativo del IGN. Expediente personal de Felipe Casares Morales.

${ }^{12}$ Memoria descriptiva del Sitio de Riofrío. José Giralt Torner, Madrid, 4 de febrero de 1869. Ms. IGN, Archivo Topográfico. Riofrío, Caja 1. 
conjunto observada por el geómetra Adolfo de Motta Francés en el Real Sitio de San Ildefonso, con el objeto de pasar el azimut y servir de comprobación a los ejes de la poligonación ${ }^{13}$ (figura 3).

Figura 3. Proyecto de triangulación y poligonación del Monte de Riofrío. Segovia. San Ildefonso ${ }^{14}$.

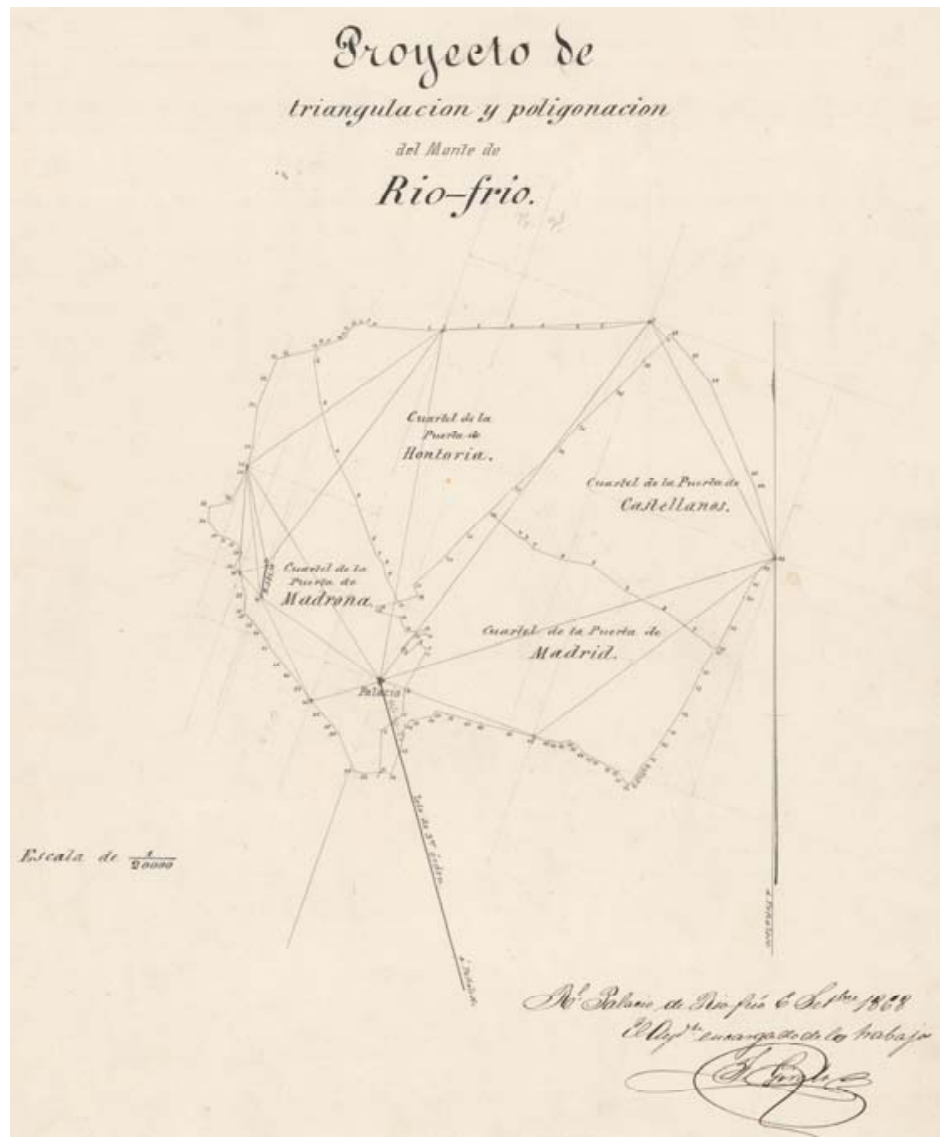

Fuente: Información geográfica propiedad del Instituto Geográfico Nacional, Archivo Topográfico, $\mathrm{n}^{\mathrm{o}}$ 400.823 .

${ }^{13}$ Poligonación de Riofrío. Observaciones horizontales y azimutales. Cuaderno $\mathrm{n}^{\mathrm{o}} 1$. Instrumento usado: Teodolito de Brunner. Observador J. Giralt Torner. Septiembre de 1868. IGN, Archivo Topográfico. Riofrío, Caja 1.

${ }^{14}$ Escala 1:20.000. Real Palacio de Riofrío, 6 de septiembre de 1868. 
En el proyecto de poligonación, que está firmado el 6 de septiembre de 1868, el bosque de Riofrío aparece dividido en cuatro "cuarteles", o grandes polígonos topográficos, denominados según las cuatro puertas del recinto que existían por entonces: la puerta de Madrona, la puerta de Hontoria, la puerta de Castellanos y la puerta de Madrid. Una semana más tarde se efectuó la compensación de los valores angulares y cierres de los triángulos, operación de la que se conserva un croquis manuscrito ${ }^{15}$. Alrededor del palacio, y de los edificios contiguos, se observó asimismo una pequeña poligonación que enlaza con la principal del perímetro ${ }^{16}$.

El propio José Giralt se encargó de elegir una base corta, para dar escala y orientación al conjunto del levantamiento. La base quedó emplazada en una zona relativamente llana, situada en el cuartel de la puerta de Madrona, relativamente cerca del Palacio. La medición de la base se reiteró seis veces, obteniendo un promedio de 181,33 metros. Todos los puntos de la poligonación y la triangulación se fijaron en los planos por las coordenadas rectangulares, referidas al meridiano y paralelo de San Ildefonso, y la orientación se obtuvo por la del lado común de la triangulación de conjunto.

Para obtener una cota de referencia se trasladó mediante nivelación directa la cota de la colegiata de la Granja de San Ildefonso, dejando una señal en el dintel de una de las puertas del palacio de Riofrío. La altitud al Mediterráneo obtenida por este procedimiento fue de $1.065,62$ metros $^{17}$. La nivelación de precisión efectuada años más tarde por el Instituto Geográfico y Estadístico asignó al mismo lugar una cota de $1.054,70$ metros, tal como consta en la placa de bronce situada en la pared del Palacio. La diferencia de $+10,92$ metros, coincide casi exactamente con la desviación del mismo signo y valor que se había producido al calcular previamente la altitud de la colegiata de San Ildefonso; su rectificación era prácticamente imposible al tratarse de un traslado de $\operatorname{cotas}^{18}$. La determinación de las curvas de nivel de cinco en cinco metros se hizo mediante el trazado de perfiles, utilizando una brújula de Gravet, cerrando polígonos de nivelación que servían de comprobación ${ }^{19}$.

Durante los meses de septiembre y octubre, y al tiempo que estaban en curso las operaciones citadas, se ejecutó el levantamiento topográfico de detalle. Los itinerarios topográficos se levantaron con brújula, siguiendo los caminos y arroyos, y fijando en

${ }^{15}$ Monte de Riofrío. Compensación. Valores angulares y cierres de los triángulos. Real Palacio de Riofrío, 13 de septiembre de 1868. Una hoja ms. de $31 \times 43 \mathrm{~cm}$. IGN, Archivo Topográfico, Riofrío. Caja 1.

${ }^{16}$ Monte de Rio-frío. Croquis de la Poligonación del Palacio. Sin fecha. Sin escala. Firmado J. Giralt. IGN, Archivo Topográfico, Riofrío. Caja 1.

${ }^{17}$ Cota al Real Palacio de Riofrío. Un cuaderno manuscrito. Sin fecha. Fdo: José Giralt. IGN, Archivo Topográfico. Riofrío, Caja 1.

${ }^{18}$ Sobre el procedimiento de nivelación del Real Sitio de San Ildefonso véase Urteaga y Camarero, 2014b.

${ }^{19}$ Itinerarios y perfiles. Término de Riofrío anejo al de San Ildefonso. Instrumento usado: Brújula de Gravet. Operadores: Giralt, Casares y Ramos. Concluido en octubre de 1868. IGN, Archivo Topográfico. Riofrío, Caja 1. 
ellos los principales elementos del terreno: puestos de caza, fuentes, ruinas, etc. En el levantamiento de los itinerarios tomaron parte los ayudantes Domingo Ramos y Felipe Casares, además del propio José Giralt.

El objeto del trabajo de detalle era, en primer término, obtener los polígonos de rústica a escala 1:2.000, con cobertura sobre todo el Real Sitio, que serían la base para el dibujo posterior de las Hojas kilométricas, el documento cartográfico que los técnicos de la Junta consideraban esencial en todo levantamiento (Muro, et al., 1996). Los polígonos de rústica se dibujaron en cuatro hojas de gran tamaño, y de formato irregular, correspondientes a cada uno de los cuatro cuarteles en que se había dividido el término. Tres de ellas, las relativas a los cuarteles de las puertas de Castellanos, Madrid y Madrona están firmadas por José Giralt y fechadas el 6 de septiembre de 1868 (figura 4).

Figura 4. Monte de Rio-frío. San Ildefonso. Cuartel de la Puerta de Madrona ${ }^{20}$.

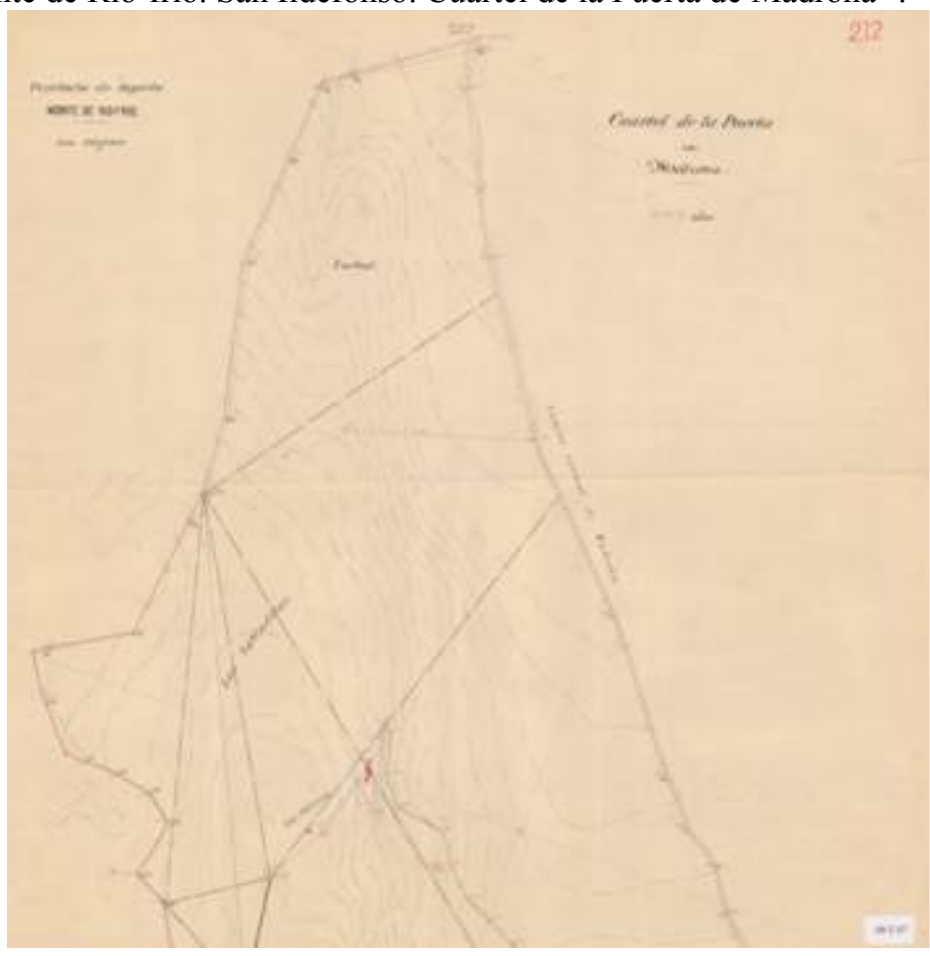

Fuente: Información geográfica propiedad del Instituto Geográfico Nacional, Archivo Topográfico, $\mathrm{n}^{\circ} 400.827$.

${ }^{20}$ Detalle. Real Palacio de Riofrío, 6 de septiembre de 1868. Escala 1:2.000. Dimensiones originales de la hoja: 75 x $150 \mathrm{~cm}$. 
El Reglamento de operaciones topográfico-parcelarias establecía además la necesidad de representar la planta de todos los edificios importantes. En consecuencia, el levantamiento de detalle incluía una labor paralela, a escala 1:500, en la que se representan las plantas de los edificios. Los edificios singulares se dibujaban aisladamente, o a veces compartiendo hoja con una construcción vecina de interés semejante. La planta baja de los edificios se presenta delimitada mediante un polígono topográfico. En el caso de Riofrío los geómetras efectuaron el levantamiento planta a planta tanto del Palacio Real como de la Casa de Oficios. En una curiosa división de tareas, Domingo Ramos se ocupó del levantamiento de las plantas bajas, mientras que Felipe Casares se ocupaba de la planta principal de los mismos edificios (figura 5). Enfrente del edificio de la Casa de Oficios, y guardando simetría respecto a la fachada principal del palacio, se había iniciado la construcción de un convento para los religiosos descalzos, que, como sabemos, había quedado paralizado. Domingo Ramos se ocupó del levantamiento de la parte construida, que estaba ya en ruinas.

Figura 5. Monte de Rio-frío. San Ildefonso. Plano de la planta principal del Palacio de Riofrío ${ }^{21}$.
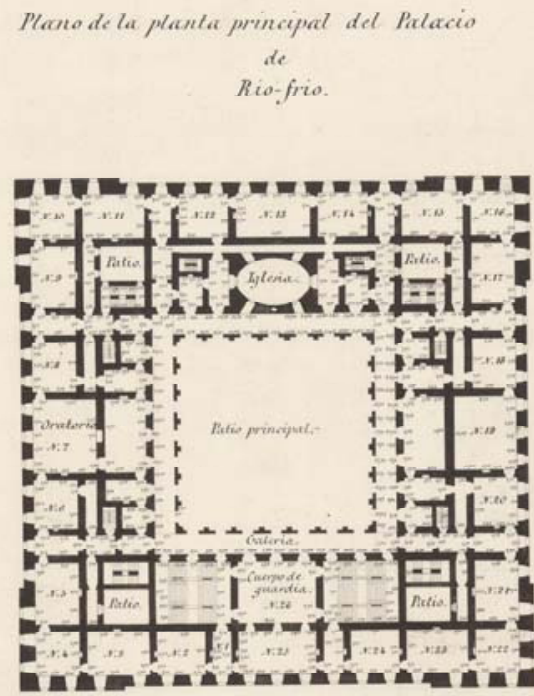

Fuente: Información geográfica propiedad del Instituto Geográfico Nacional, Archivo Topográfico, $\mathrm{n}^{\mathrm{o}}$ 400.819 .

${ }^{21}$ Escala 1:500. Detalle. Dimensiones originales de la hoja: 30 x $46 \mathrm{~cm}$. El Ayudante Geómetra Felipe Casares. Revisado, el Ayudante encargado de los trabajos: J. Giralt. Firmado en Toledo el 22 de diciembre de 1868. 


\section{TRABAJOS DE GABINETE}

Finalizada la campaña de trabajos de campo, José Giralt Torner regresó a Madrid para acometer las labores de gabinete. Su primera tarea fue realizar los cálculos definitivos de la triangulación y la poligonación, labor que dio por concluida el 16 de diciembre de $1868^{22}$. Sin embargo, había llegado a la capital con la salud quebrantada. El 15 de diciembre solicitó licencia de un mes para reponerse. Previo informe de Ventura Pizcueta Chirivella, asegurando la veracidad de su dolencia, se le concedió permiso para trasladarse a Barcelona. Regresó a Madrid el 26 de enero de 1869, justo a tiempo para finalizar las tareas de gabinete ${ }^{23}$. En las labores de dibujo contó con el auxilio del delineante José Pilar Morales, uno de los dibujantes más veteranos de la Junta de Estadística.

José Pilar Morales Ramírez había nacido en Santa Cruz de Mudela (Ciudad Real) en 1823. Se formó en la Escuela de Arquitectura de Madrid, donde llegó a aprobar dos cursos. Posteriormente obtuvo los títulos de maestro de obras y director de caminos vecinales por la Real Academia de San Fernando (Madrid). En 1849 se incorporó a los talleres del Atlas de España, que dirigía Francisco Coello, donde permaneció hasta 1860 alcanzando el puesto de encargado de los talleres de grabado y litografía. En agosto de 1860 solicitó una plaza del personal facultativo para los trabajos geográficos en la Comisión de Estadística General de Reino, acompañando la solicitud con una calurosa carta de recomendación del teniente coronel de Ingenieros, Francisco Coello Quesada, por entonces vocal de la Comisión de Estadística. El 29 de enero de 1861 fue nombrado delineante de la Comisión de Estadística General del Reino. Cinco años más tarde alcanzó el cargo de primer delineante de la Dirección de Operaciones Geográficas, con un salario de 12.000 reales anuales. Una de las últimas tareas en las que tomó parte fue precisamente el dibujo y encaje de los planos correspondientes al Real bosque de Riofrío ${ }^{24}$.

Los trabajos de gabinete se prolongaron hasta mediados de febrero de 1869, dando lugar a un conjunto de 28 planos que se ajustan estrictamente a la normativa prevista en el Reglamento de Operaciones Topográfico-Catastrales aprobado en 1865. En primer término se dibujó un plano de conjunto, a escala 1:20.000, que sirve como plano director del Real Sitio, en el que se señalan los puntos de la triangulación principal y las estaciones observadas en el perímetro de la finca. En el margen iz-

\footnotetext{
${ }^{22}$ Poligonación de Riofrío. Cálculo. Cuaderno no 1 que contiene 12 polígonos calculados por D. José Giralt Torner. Madrid, 16 de diciembre de 1868. IGN, Archivo Topográfico. Riofrío, Caja 1.

${ }^{23} \mathrm{Su}$ salud, sin embargo, se había resentido de modo irremediable. Falleció el 20 de septiembre de 1870, con tan sólo 28 años de edad.

${ }_{24}$ El 9 de marzo de 1869 , apenas concluida la tarea a la que nos referimos, fue declarado cesante en la Junta General de Estadística por supresión de la sección de dibujo. A partir de 1870 continuó ejerciendo el oficio de grabador en el ámbito privado. Fuente: Archivo histórico y administrativo del IGN. Expediente personal de José Pilar Morales Ramírez.
} 
quierdo, y a doble columna, se consignan las tres coordenadas de los puntos fijados por procedimientos trigonométricos: en este caso un total de 120.

Relacionados con este plano de conjunto, y con las cuadrículas trazadas en él, se presentan los detalles topográficos del Real Sitio en hojas cuadriculadas a escala 1:2.000. En total, el parcelario rústico comprende trece hojas kilométricas a la escala citada. Cada una de las hojas recoge los vértices de la triangulación, los cursos de agua, los caminos y sendas, las curvas de nivel de cinco en cinco metros, y la toponimia (figura 6). Excepcionalmente, en el caso de Riofrío, el conjunto de hojas kilométricas se redujo en un plano a escala 10.000, que permite apreciar una visión general de Riofrío, sin perder los detalles del terreno.

Figura 6. Provincia de Segovia. Rio-frío. San Ildefonso ${ }^{25}$.

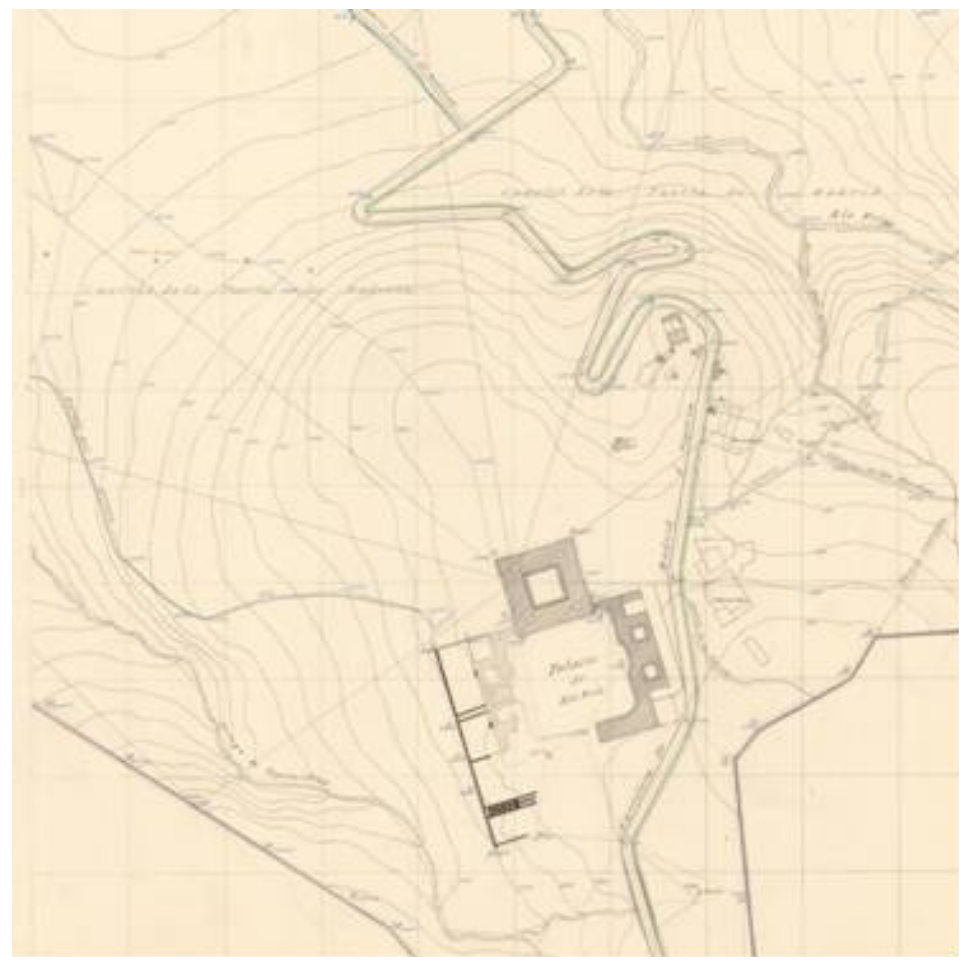

Fuente: Información geográfica propiedad del Instituto Geográfico Nacional, Archivo Topográfico, $\mathrm{n}^{\circ} 400.841$.

${ }^{25}$ Escala 1:2.000. Hoja kilométrica 2C. Detalle. Ejemplo de una de las trece hojas kilométricas que comprende el término de Riofrío. Dimensiones originales de la hoja: 85 x $60 \mathrm{~cm}$. 
Figura 7. Topografía Catastral de España ${ }^{26}$.

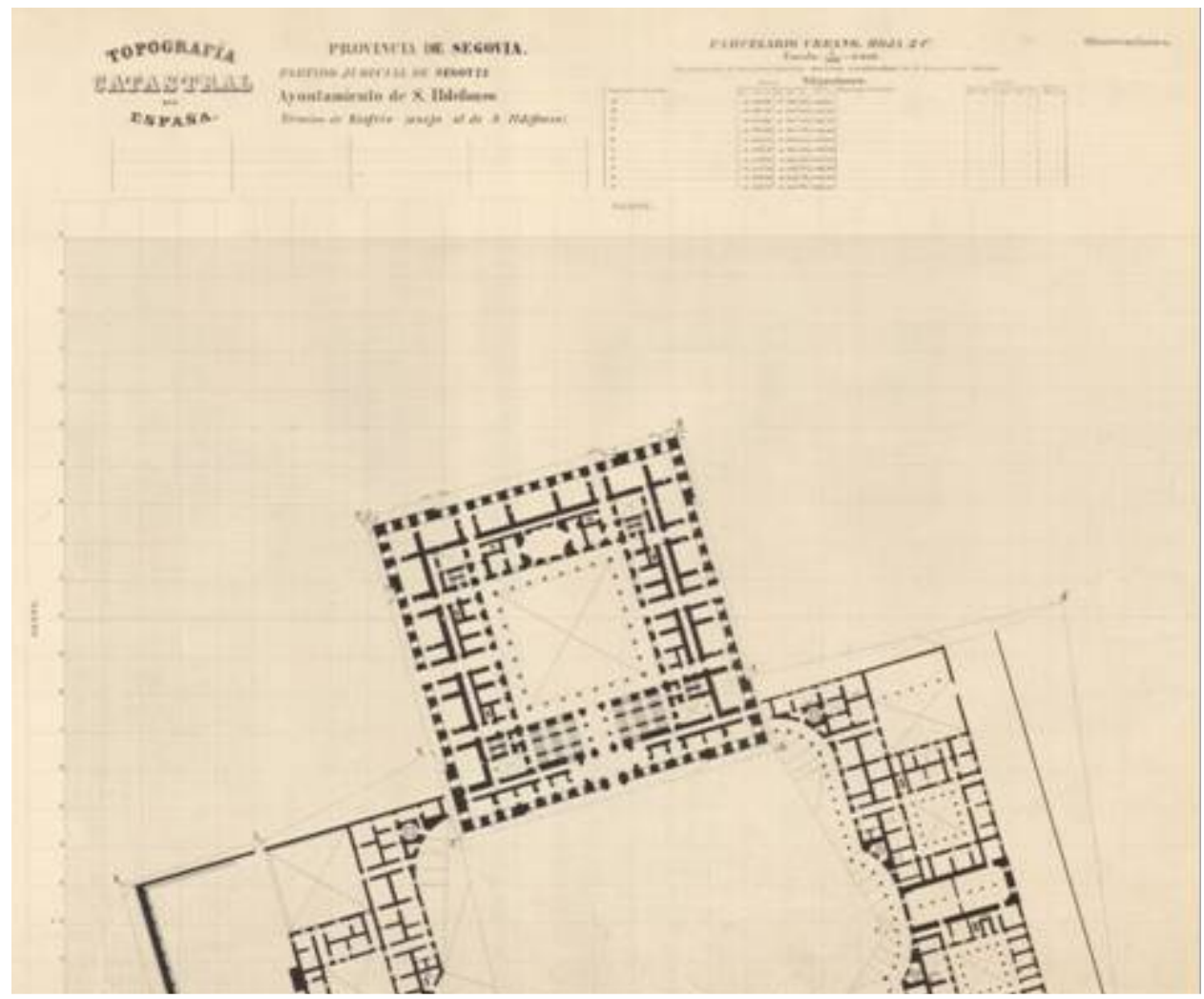

Fuente: Información geográfica propiedad del Instituto Geográfico Nacional, Archivo Topográfico, ${ }^{\circ}$ 400.831 .

Por último, José Giralt y José Pilar Morales procedieron al dibujo definitivo de los planos a escala 1:500, que recibieron el nombre, bastante inapropiado en este caso, de "Parcelario urbano". Las diferencias esenciales entre las minutas y el dibujo definitivo son cuatro: primero, el "Parcelario Urbano" se dibuja sobre papel normalizado y cuadriculado en hojas de $70 \times 60 \mathrm{~cm}$, con una mancha de $60 \times 40 \mathrm{~cm}$; segundo, todas las hojas incluyen en su parte superior una cartela que identifica la colección cartográfica y el territorio representado, con denominación de la provincia, partido judicial y término municipal; tercero, los planos parcelarios quedan encajados en la red de coordenadas planas definida por la Junta de Estadística, a la que quedan

${ }^{26}$ Ayuntamiento de San Ildefonso. Término de Riofrío (anejo al de San Ildefonso). Parcelario urbano. Hoja 2C. Escala 1:500. Dimensiones de la hoja: 76 x $59 \mathrm{~cm}$. 
referidos mediante un número y una letra; y cuarto, los planos están orientados al norte geográfico (figura 7).

\section{CONCLUSIONES}

Entre agosto de 1868 y febrero de 1869 un equipo de geómetras de la Junta General de Estadística, dirigido por José Giralt Torner, realizó los trabajos de campo y gabinete necesarios para proceder a una representación cartográfica fiable del Real Sitio de Riofrío. El levantamiento formaba parte de un proyecto cartográfico más ambicioso, que tenía por objeto el inventario y deslinde del patrimonio territorial de la Corona. El levantamiento se inició en plena agonía del régimen isabelino. Cuando concluyó, la reina Isabel II estaba en el exilio, y los bienes cartografiados se consideraban integrantes del Patrimonio nacional. Así lo refleja la primera frase del informe final de Giralt Torner, librado el 4 de febrero de 1869: "Este sitio es propiedad de la Nación".

Los planos formados por los geómetras de la Junta de Estadística, que se conservan inéditos en el archivo del Instituto Geográfico Nacional, constituyen un conjunto documental de notable valor geohistório, sin posible parangón entre las fuentes iconográficas del siglo XIX. El citado fondo documental consta de 28 mapas a gran escala (entre 1:500 y 1:20.000), y un número similar de croquis y dibujos preparatorios. También se conservan los cuadernos de campo y las memorias y cálculos correspondientes a la poligonación y nivelación del término.

Las tareas catastrales de la Junta General de Estadística quedaron bruscamente interrumpidas en el otoño de 1869 , al procederse a la reorganización de los servicios cartográficos del estado. Como consecuencia de esta interrupción, el conjunto de planos procedentes del levantamiento de los Sitios Reales, y en particular los correspondientes al Real Sitio de Riofrío, fueron archivados y quedaron en el olvido.

\section{BIBLIOGRAFÍA}

BREÑOSA, R. y CASTELLARNAU, J. M. (1884): Guía y descripción del Real Sitio de San Ildefonso. Madrid, Tipografía de los Sucesores de Rivadeneyra.

CAMARERO BULLÓN, C., FERRER RODRÍGUEZ, A. y NIETO CALMAESTRA, J. A. (2012): Cartografía parcelaria urbana de Granada: levantamiento topográficoparcelarios de la Junta Generalde Estadística. CT Catastro, 74, $27-58$.

COS-GAYÓN, F. (1881): Historia jurídica del patrimonio real. Madrid, Imprenta de Enrique de la Riva.

HERNANDO CORDERO, J. F. (1995): Historia de la construcción del Palacio de Riofrío. Reales Sitios, 126, 43-53.

HERNANDO CORDERO, J. F. (1997): El Real bosque de Riofrío. Reales Sitios, 132, 2-13. 
HERNANDO CORDERO, J. F. (2008): El Sitio Real de Riofrío. Historia de un proyecto cortesano en la España del siglo XVIII. Tesis doctoral. Universidad de León, Facultad de Filosofía y Letras.

HERNANDO CORDERO, J. F. (2009): Los diseños del Sitio Real de Riofrío en la colección Rabaglio. Reales Sitios, 181, 42-59.

HERNANDO CORDERO, J. F. (2010): Las edificaciones cortesanas del Real Sitio de Riofrío. De Arte, 9, 121-138.

LAVALLE COBO, M. T. (1994): El mecenazgo de Isabel de Farnesio, Reina de España. Tesis doctoral. Universidad Autónoma de Madrid.

LAVALLE COBO, M. T. (2002): Isabel de Farnesio. La Reina coleccionista. Madrid, Doce Calles.

MURO, J. I., NADAL, F. y URTEAGA, L. (1996): Geografía, estadística y catastro en España, 1856-1870. Barcelona, Ediciones del Serbal.

SANCHO, J. L. (1995): La arquitectura de los Sitios Reales. Catálogo histórico de los Palacios, Jardines y Patronatos Reales del Patrimonio Nacional. Madrid, Patrimonio Nacional.

SANCHO, J. L. y APARICIO, J. R. (2012): Real Sitio de la Granja de San Ildefonso y Riofrío. Madrid, Patrimonio Nacional.

URTEAGA, L. (2011): El profesorado de la Escuela del Catastro. CT Catastro, 71, 29-53.

URTEAGA, L. y CAMARERO BULLÓN, C. (2014a): Los planos de los Reales Sitios españoles de la Junta General de Estadística, 1861-1869. Scripta Nova, (en publicación).

URTEAGA, L. y CAMARERO BULLÓN, C. (2014b): Planimetría del Real Sitio de San Ildefonso de la Junta General de Estadística, 1868-1869. Treballs de la Societat Catalana de Geografia, 77 (en publicación). 